\title{
Audiovisuales didácticos para promover las competencias científicas en la educación a distancia
}

1. Encargada de la Cátedra de Ciencias Naturales, Escuela de Ciencias Exactas y Naturales,

UNED, Costa Rica; marodriguez@uned.ac.cr

2. Productora audiovisual, Programa de Producción de Material Audiovisual,

UNED, Costa Rica; ivillalobos@uned.ac.cr

Recibido: 22 de mayo del 2017

Corregido: 03 de julio del 2017

Aceptado: 20 de julio del 2017

\begin{abstract}
Resumen
Como estrategia metodológica, la Cátedra de Ciencias Naturales perteneciente a la carrera de Manejo de Recursos Naturales y el Programa de Producción de Material Audiovisual de la Universidad Estatal a Distancia produjeron dos videos didácticos, Especies Marinas: Caribe Submarino y Especies Marinas: Acuario Marino, para ser utilizados en la asignatura Laboratorio de Zoología General. Al ser una asignatura impartida en el tercer bloque del plan de estudios de la carrera, se espera que desde etapas tempranas los estudiantes accedan a estrategias pedagógicas abiertas, flexibles y dinámicas que consideren la diversidad de formas, estilos y ritmos de aprendizaje en procura de que el mismo sea significativo, inclusivo y participativo mediante el uso de los recursos audiovisuales.

Los dos videos didácticos deben ser observados y analizados por los estudiantes antes de realizar la gira de la asignatura Laboratorio de Zoología General, la cual forma parte de la evaluación de los estudiantes. La expectativa es que, a largo plazo, los estudiantes desarrollen competencias de pensamiento científico a partir de explorar una gran variedad de habilidades científicas tales como: observación, experimentación, manipulación, argumentación y expresión cuando están ejecutando la práctica de campo. Por lo tanto, en estos audiovisuales se asocian los conocimientos teóricos y prácticos de varios contenidos de la asignatura, para que posteriormente ellos puedan investigar, analizar e interpretar en procura de un pensamiento científico más activo y crítico.

En ambas producciones, la estrategia narrativa reúne diversos recursos audiovisuales con fines didácticos, entre ellos: video, video submarino y animación que describen las diversas etapas del proceso y el entorno, por ello el uso de videos didácticos forma parte de una metodología pedagógica para promover el desarrollo de las competencias científicas y lograr un cambio positivo y dinámico en el aprendizaje de los estudiantes.
\end{abstract}

Palabras clave: Videos didácticos, Competencias científicas, Laboratorio de Zoología general, Especies Marinas, Educación a distancia.

\section{Abstract \\ Didactic audiovisuals to promote scientific skills in distance education}

As a methodological and as an strategy of evaluation, the Professorship of Natural Sciences, part of the Natural Resources Management program of the State University at Distance, and in collaboration with the Audiovisual Program, two didactic videos were elaborated: "Marine Species: Caribbean Submarine" and "Marine Species: Marine Aquarium", to be used in the subject of General Zoology Laboratory. Being this subject taught in the third quarter of the study plan of the career, it is expected 
that from the early stages students access open, flexible and dynamic pedagogical strategies that consider the diversity of teaching forms, styles and rhythms in search of learning significant, inclusive and participative with the use of audiovisuals resources.

After observing and analyzing the two didactic videos by the students before performing the field tour of the subject of General Zoology Laboratory, which is part of the evaluation of students. The expectation is that long-term students develop scientific thinking skills from exploring a great variety of scientific skills such as: observation, experimentation, manipulation, argumentation and expression, when they are executing the field practice. In these audiovisuals, therefore, the theoretical and practical knowledge that is developed of some of the contents of the subject is associated, so that they can later investigate, analyze and interpret in search of a more active and critical scientific thought.

In both productions, the narrative strategy brings together various resources audiovisual for didactic purposes, among them: video, underwater video and animation that describes the various stages of the process and the environment, that is why the use of didactic videos is part of a pedagogical methodology, to promote the development of scientific competences and achieve a positive and dynamic change in student learning.

Key words: Didactic videos, Scientific competences, General Zoology Laboratory, Marine Species, Distance education.

\section{INTRODUCCIÓN}

Todo modelo educativo, principalmente la educación a distancia, se enfrenta al reto y a la oportunidad de construir procesos de mediación pedagógica que favorezcan el aprendizaje independiente, crítico y continúo de los estudiantes.

Para la Universidad Estatal a Distancia de Costa Rica (UNED), el desarrollar competencias científicas en el área de las ciencias naturales es un eje clave para promover la utilización de diversas técnicas y herramientas en los estudiantes, para que cuando se tengan que enfrentar al entorno puedan apropiarse de sus destrezas y habilidades de manera dinámica, crítica y creativa propiciando un proceso de aprendizaje significativo.

Tomando en cuenta estos factores se produjeron los audiovisuales didácticos Especies Marinas: Caribe Submarino y Especies Marinas: Acuario Marino, utilizados en la asignatura Laboratorio de Zoología General de la carrera Manejo de Recursos Naturales (MARENA), con los cuales se busca fortalecer habilidades científicas como observación, experimentación, manipulación, argumentación y expresión.

La documentación de la experiencia de producción a nivel metodológico y los resultados de su validación con estudiantes de la asignatura tiene por objetivo mostrar una estrategia pedagógica que, mediante la producción y uso de audiovisuales didácticos, busca promover habilidades científicas que a largo plazo contribuyan al desarrollo de las competencias de pensamiento científico en los estudiantes y futuros profesionales de las ciencias naturales.

Ambos videos didácticos derivaron de un diálogo de saberes, entre docentes de las ciencias y productores audiovisuales de la UNED. Por lo tanto, es recomendable que este procedimiento se realice como una práctica constante en procura de garantizar la rigurosidad científica y narrativa de los materiales audiovisuales didácticos. 


\section{Desarrollo de competencias de pensamiento científico mediante la docencia}

La Zoología es considerada como una rama de la biología y según Ravanal (2012), en los últimos años muchos profesionales en el área de las ciencias, están convencidos que la enseñanza y aprendizaje en la biología debe de estar ligada al desarrollo de competencias de pensamiento científico, donde se tome en cuenta que la participación de las personas sea complementada con temas socio científicos propios de una sociedad en cambio permanente, para lograr afrontar desafíos, conflictos o problemas.

En este contexto, el pensamiento de Paulo Freire (2000) sigue vigente. El autor promueve superar la visión de la educación bancaria en la que el educador deposita un contenido programado en el educando, y en su lugar se promueve un pensamiento crítico y versátil que le permite al estudiante afrontar la diversidad de escenarios y desafíos de su proceso educativo y profesional.

Por consiguiente, para la parte práctica de la asignatura de Zoología General, perteneciente a la cátedra de Ciencias Naturales de la UNED, se utilizan diferentes estrategias metodológicas y de evaluación para lograr un adecuado proceso de enseñanza y aprendizaje en los estudiantes de la Carrera de Manejo de Recursos Naturales (MARENA).

Al considerarse la zoología como una rama de la biología, se logra ampliar los conocimientos en los estudiantes y se posibilita la exploración en una gran diversidad de áreas, entre las cuales están las investigativas, ambientales y sociales, logrando así integrar sus competencias científicas.

Por tanto, el uso de audiovisuales didácticos forma parte de una estrategia metodológica que se complementa con el pensamiento de las competencias científicas para lograr un cambio de forma positiva y dinámica en el aprendizaje de los estudiantes.

Según Angulo (2012), en la actualidad, el estado de la enseñanza de las ciencias se encuentra definido de la siguiente forma.

Ante currículos abiertos, que le exigen al profesor tomar decisiones sobre qué ciencia enseñar, cómo y cuándo hacerlo, por qué razones o para atender cuáles objetivos, y estas decisiones van acompañadas de que él/ella considera que es la ciencia, su aprendizaje y su evaluación. A su vez, tales decisiones exigen de parte del profesor, una crítica sobre lo que sabe (o cree saber) de la ciencia, de la enseñanza de las ciencias, así como de su actuación en el aula y la autoevaluación de la consistencia entre estos dos aspectos, en beneficio del aprendizaje de sus alumnos. (p.127)

Por lo tanto, se requiere que los profesores comprendan el propósito de enseñar ciencias, donde ellos enseñen a pensar, para que los estudiantes consideren los hechos o situaciones como un modelo que les permita interpretar y participar del medio que los rodea. Así, para la asignatura Laboratorio de Zoología General se pretende que los videos didácticos realizados expliquen conceptos y técnicas útiles para el desarrollo del trabajo de campo y que junto a la diversidad de materiales que componen la Unidad Didáctica Modular y otras estrategias incluidas en el diseño curricular, los estudiantes logren aprender, explicar e interpretar los hechos que se les presentan como actividades de la asignatura.

Según Martínez et al. (2001, citados por Ravanal, 2012) los profesores que enseñan Biología o ramas de ella, deben plantearse la necesidad de incorporar problemas relativos al diseño curricular, lo cual permita avanzar hacia un profesor autónomo, reflexivo, crítico e investigador, con el propósito de facilitar el aprendizaje de la ciencia y revelador de los procesos de enseñanza - aprendizaje - evaluación. 
Para Ravanal (2012):

El profesor que enseña y aprende biología es más que "sumar conocimiento" ya que exige del estudiante protagonismo cognitivo, que deriva en un aprender a aprender; esto implica entonces, identificar obstáculos y potencialidades, evaluarse para la toma de decisiones, saber comunicar y comunicarse, y teorizar para interpretar los hechos del mundo. (p.119)

Por lo tanto, es importante que los profesores sean profesionales que comprendan y promuevan competencias del pensamiento científico en los estudiantes mediante valores, actitudes, destrezas, habilidades, procedimientos y emociones que principien la búsqueda de soluciones a los problemas que se presentan en el entorno.

Para Quintanilla (2006), las Competencias del Pensamiento Científico (CPC) se refieren al "despliegue de saberes, de capacidades para afrontar una situación, de un cierto grado de dominio de habilidades y recursos, atributos sustentados en las acciones de captar, pensar, explorar, atender, percibir, formular, manipular e introducir cambios que permitan realizar una interacción competente" (p.27).

Mientras que los autores Díaz, Quintanilla y Labarrere (2012), manifiestan que se requiere promover sujetos competentes y para lograrlo indican:

Se requiere de comunidades de pensamiento y actuación, donde el grupo actúa como comunidad generadora de conocimientos y realiza actividades científicas, tendientes a la conformación de Competencias de Pensamiento Científico (CPC). Donde se configura conceptualmente a la competencia científica, como una combinación dinámica de atributos en relación a conocimientos, habilidades, actitudes, valores y responsabilidades. Esta se expresa en los resultados de aprendizaje, evidenciando que los estudiantes han aprendido a comprender la ciencia, por medio de las actividades de leer, escribir, pensar, explorar, captar, formular, percibir, atender, manipular, comunicar y trasferir conocimiento científico, de manera ágil y productiva, cuando abordan situaciones problemáticas. (p.84)

El pensamiento anterior concuerda con lo que se realiza en la asignatura de Laboratorio de Zoología General, especialmente en el desarrollo de las prácticas de laboratorio y las giras de campo donde se utilizan estrategias metodológicas para la ejecución y desarrollo óptimo de los contenidos y se realizan actividades grupales que incentivan el análisis, la criticidad, la colaboración y el trabajo interdisciplinario para integrar las destrezas, habilidades, actitudes y capacidades de cada estudiante. Así, de forma colaborativa, se promueve el desarrollo de competencias que le permitirá fortalecer al estudiante su pensamiento científico y profesional, a su propio ritmo con una visión de proceso de enseñanza y aprendizaje continuo.

Parte de los objetivos de la Universidad Estatal a Distancia como institución de educación superior a distancia es que los estudiantes sean más independientes y autodidactas en su proceso de aprendizaje. El Modelo Pedagógico de la UNED (2004) indica: "El estudiante a distancia deberá desarrollar la capacidad de no esperar pasivamente; debe saber a quién acudir para contar con apoyo no solamente referido a los contenidos y su comprensión, sino también a las estrategias y particularidades del estudio independiente y autodirigido" (p. 15).

Lo indicado en el Modelo Pedagógico de la UNED muestra relación con el desarrollo de competencias de pensamiento científico, donde se pretende que los conocimientos sean visualizados epistemológicamente sobre la ciencia y el modo en que se entiende su enseñanza. Así, se procura y promueve un aprendizaje flexible, dinámico y autorregulado, como una meta a lo largo de la vida profesional, por eso la importancia de utilizar material audiovisual en la asignatura, ya que contribuye a interiorizar mejor 
algunos conceptos, contenidos y a entender procedimientos teóricos-prácticos de la zoología de una forma más flexible y menos afanosa para su autoaprendizaje.

Según Díaz, Quintanilla y Laberre (2012), lo que se requiere en los centros educativos son estudiantes que logren una elaboración de saberes, como pensadores activos que desarrollan un sistema de pensamiento mediante un ejercicio intelectual y social, con el fin de encontrar sus propias soluciones al problema.

El desarrollo de competencias científicas no depende únicamente de un curso o asignatura particular, se requiere de todo un proceso metacognitivo y años para interiorizarlo, por lo que debe ser un proceso constante en estudiantes, docentes, productores audiovisuales y toda la institución educativa.

\section{Audiovisuales didácticos: sus características y aportes}

Buckingham y Scanlon (2003), en su libro Education, Entertainment and Learning in the Home, explican y ejemplifican el creciente interés en el potencial de aprender desde la casa tendiente a borrar los límites entre la casa y la escuela, los padres y los maestros, entre lo público y lo privado y entre la educación y el entretenimiento donde los nuevos medios (Internet, CD, DVD) cumplen un rol fundamental y toman cada vez más auge como herramientas didácticas de fácil acceso.

Por tanto, la producción audiovisual didáctica cobra cada vez más relevancia en los hábitos de consumo de las nuevas generaciones y sobre todo aquellas que estudian en el modelo de educación a distancia. Como lo explican los autores Buckingham y Scanlon:

La mayoría de los niños que tenían la opción dijo que preferirían usar nuevos medios (Internet o CD-ROM) en lugar de libros para encontrar información. Cuando se les dio tarea que involucraba alguna investigación, su primer instinto fue comprobar en la computadora, en lugar de usar un libro. (2003, pp. 179-180)

Ante la creciente apropiación de los audiovisuales, como parte de los hábitos de consumo de la población en general y de los estudiantes, es necesario aclarar que existen características diferenciadoras entre una producción televisiva comercial y un video didáctico producto de una mediación pedagógica consciente de los objetivos que motivan su realización.

Cebrián de la Serna (1994) define y caracteriza el video didáctico desde una circunstancia particular "que esté diseñado, producido, experimentado y evaluado para ser insertado en un proceso concreto de enseñanza aprendizaje de forma creativa y dinámica" (p. 15). Este es el caso de los videos didácticos Especies Marinas.

Aunado a lo anterior, se deben de tomar en cuenta algunas características clave para comprender los videos didácticos. Cebrián de la Serna (1994) a lo largo de su publicación Los videos didácticos: claves para su producción y evaluación destaca que estos deben estar integrados con otros recursos didácticos (el libro, las orientaciones de curso, las prácticas de campo, etc.), deben contar con una guía para su uso (momento del visionado y tareas específicas por realizar a partir de ellos), deben conjugar los diversos recursos narrativos del lenguaje audiovisual, logrando un equilibrio entre contenido racional y emotividad que conduzca a lo que él llama "la emoción por el conocimiento" y un aspecto sumamente importante es que no existe una fórmula para definir el tiempo o duración más adecuada; pero se recomiendan duraciones concretas entre los 5 minutos y los 20 minutos.

Otros autores comentan sobre aspectos de importancia que conviene tomar en cuenta en los audiovisuales, como es el caso de Ruíz, Sánchez y Maroto (s.f.), quienes indican que los audiovisuales emplean principalmente el recurso de video y audio, pero cuando se seleccionan como apoyo de material 
didáctico requieren tomar aspectos de mediación pedagógica, "siempre se deben de producir o elegir los que, aprovechando el lenguaje audiovisual de la imagen, el sonido y el texto, provoquen en los estudiantes procesos de autoaprendizaje y reflexiones sobre las temáticas tratadas, para motivar procesos cognitivos de niveles superior" (p.126).

Para Peters (2013, citado por Ruíz, Sánchez y Maroto, s.f.), el video es especial porque presenta un atractivo único con su combinación de luz, sonido y color. Además, debido a que permite ver cómo suceden las cosas de forma realista, ya sea una transmisión de noticias en vivo o un procedimiento médico, se pueden atestiguar muchos detalles. De esta forma, las historias contenidas en un video pueden lograr un efecto profundamente emocional en las personas.

Un estudio sobre educación a distancia realizado en España por los autores Arias y Venegas (2013), demostró que los videos son los recursos favoritos de consulta para los estudiantes, siendo los documentos de texto la segunda opción.

Por lo tanto, el uso de materiales didácticos en formato audiovisual en la asignatura Laboratorio de Zoología en la UNED es de suma importancia por ser un medio que al conjugar los contenidos teóricos y prácticos favorece el desarrollo de competencias científicas.

\section{Ruta de producción para la elaboración de los videos didácticos Especies Marinas}

Para la producción de los videos didácticos Especies Marinas en la asignatura Laboratorio de Zoología General fue necesario un diálogo de saberes entre docente y equipo de producción; directora, camarógrafo, editor y animador, el cual condujera a un proceso de traducción, que como explica De Sousa (2009):

Es el procedimiento que permite crear inteligibilidad recíproca entre las experiencias del mundo, tanto las disponibles como las posibles (...) se trata de un procedimiento que no atribuye a ningún conjunto de experiencias ni el estatuto de totalidad exclusiva ni el estatuto de parte homogénea. (p. 136)

De acuerdo con lo anterior, de manera conjunta se tomaron en consideración las necesidades pedagógicas detectadas por la docente que administra la asignatura y las características y potencialidades del lenguaje audiovisual para diseñar la propuesta de producción. Se tomaron en cuenta criterios como los siguientes:

a. Ubicación de la asignatura en el plan de estudios: programada para el tercer bloque del plan de estudios del bachillerato de la carrera de MARENA, de ahí la importancia de incorporar en etapas tempranas las estrategias metodológicas que procuren el desarrollo de competencias científicas y habilidades como la observación, la experimentación, la interpretación de resultados, el análisis, la argumentación y la expresión.

b. Necesidades didácticas prioritarias de la asignatura: una de las actividades prácticas de la asignatura consiste en una gira de campo que incluye dos grandes tareas: la interpretación ambiental de senderos y la búsqueda, identificación y caracterización de organismos marinos. Esta última requiere poner en práctica una guía de campo minuciosa en condiciones ambientales complejas, como lo es el área de plataforma continental en el Refugio de Vida Silvestre Gandoca Manzanillo, ubicado en la provincia de Limón. Los estudiantes desarrollan el trabajo de campo en el mar, lo cual exige tomar en cuenta una serie de protocolos de seguridad como por ejemplo vestimenta y equipo adecuado que les permita a los estudiantes movilizarse en el agua para identificar y manipular los 
especímenes de manera correcta sin alterar significativamente el ecosistema. La experiencia de los docentes de la asignatura identificó que los estudiantes llegaban a la práctica de campo con muchas dudas y ansiedad.

Con base en lo anterior y la revisión bibliográfica, se determinó que por practicidad y uso de los estudiantes se requerían dos videos, Especies Marinas: Caribe Submarino (15 minutos de duración) enfocado en el trabajo de campo en plataforma continental y Especies Marinas: Acuario Marino (10 minutos de duración) que guía la actividad en el acuario marino.

El primer video aborda los siguientes contenidos: materiales para el trabajo de campo (vestimenta y equipo), metodología para el desarrollo de las actividades (selección del cuadrante, captura e identificación de especies marinas) y completar la bitácora o guía de trabajo de campo. El segundo aborda los siguientes contenidos: metodología de incorporación de especies al acuario marino, mantenimiento y necesidades de los especímenes dentro del nuevo entorno, protocolos de alimentación, filtración, iluminación, control de PH y sales.

Para cada uno de los videos didácticos se elaboró en primera instancia una escaleta, es decir un documento escrito que desglosa los principales contenidos que requerían ser desarrollados o expuestos. Seguidamente, se pasó a la escritura de guiones donde se define cómo se quiere presentar lo que se necesita contar. Explica Syd Field (1996) "Un guion sigue una línea de acción narrativa determinada, concisa y ajustada, una línea de desarrollo" (p.14).

Para el caso de los videos didácticos Especies Marinas primero se escribió el guion literario, es decir un texto que incluye las explicaciones teóricas, metodológicas, prácticas y de análisis que faciliten la comprensión de los contenidos seleccionados previamente en la escaleta. Posteriormente se elaboró el guion técnico, el cual detalla el tipo de imágenes, sonidos, música, animación y entrevistas que permiten mostrar a nivel audiovisual los conceptos y los procesos; este último guion es una puesta por escrito de lo que finalmente el estudiante podrá ver, pues todo se planifica en esta etapa.

En ambas producciones, la estrategia narrativa reúne diversas herramientas audiovisuales con fines didácticos, entre ellas: video, video submarino y animación hiladas mediante un narrador en off que describe las diversas etapas del proceso y el entorno, todas las etapas y decisiones son minuciosamente revisados entre productores audiovisuales y especialistas de contenidos del área. Con ello se procura una estrategia pedagógica abierta, flexible y dinámica, que considera la diversidad de formas, estilos y ritmos de aprendizaje en procura de que el mismo sea significativo, inclusivo y participativo.

Los audiovisuales asocian los conocimientos teóricos con los prácticos que posteriormente ellos y ellas mismas podrán explorar, manipular e interpretar en procura de un pensamiento científico más productivo. A su vez, ambos videos contribuyen con la puesta en práctica de sus habilidades científicas en la búsqueda de un empoderamiento paulatino de las competencias del pensamiento científico.

Los videos se pusieron a disposición en el portal de audiovisuales de la UNED que se encuentra en el siguiente enlace http://audiovisuales.uned.ac.cr/mediateca/videos y posteriormente se cargaron en el canal de Youtube Audiovisuales UNED.

Ambos videos debían observarse previo a cada actividad, de tal forma que les permitiera a los estudiantes abordar de manera individual y colectiva el trabajo de campo para posteriormente presentar un informe de gira o trabajo de campo como parte de los instrumentos de evaluación en la asignatura. 


\section{MATERIALES Y MÉTODOS}

La metodología aplicada para la validación de los videos didácticos incluyó elementos de tipo cualitativo y cuantitativo. El primero desarrrolla la observación no participante que como explica Flick (2007) "se abstiene de intervenciones en el campo, a diferencia de las entrevistas y las observaciones participantes" (p.150), mientras el segundo se enfocó en la formulación de un instrumento de validación o cuestionario.

El instrumento de validación se tituló Consulta a Estudiantes de la Carrera Manejo de Recursos Naturales sobre el uso y el aporte de los Audiovisuales: Especies Marinas: Acuario Marino y Especies Marinas: Caribe Submarino; se le entregó de manera impresa a cada estudiante posterior a la práctica de campo, fue autoadministrado, debían responder directamente en el documento y los datos obtenidos fueron trasladados al programa Excel donde fueron procesados.

El instrumento contempló preguntas cerradas y semiabiertas, cada una de ellas responde a una variable de interés a nivel de contenidos del curso y objetivos de producción por los cuales se realizaron los videos y por tanto era prioritario conocer el criterio de los estudiantes. El cuestionario contó con 17 preguntas en total y de acuerdo con lo requerido se establecieron los valores, escala de medida y categorías. Primero se consultó información sobre edad e identidad de género con el objetivo de lograr un perfil general del estudiante. Posteriormente, se presentaron siete preguntas cerradas de selección única y respuesta dicotómica (sí o no) para los contenidos más concretos como: observación de los videos y pertinencia de los contenidos expuestos en general.

Adicionalmente, se aplicaron siete preguntas cerradas de escala de medición que como explican Palella y Martins (2012) "las escalas son formas de medida que se basan en la idea de clasificación, aprovechando a la par las propiedades semánticas de las palabras" (p.136). En el cuestionario que completaron los estudiantes se empleó la escala de valores (muy clara, clara, poca clara y deficiente) para el abordaje de contenidos más puntuales sobre el desarrollo del trabajo de campo expuesto en los videos. Finalmente, se presentaron tres preguntas abiertas de clarificación con espacio para que los estudiantes indicaran recomendaciones y sugerencias pues en las mismas "el usuario aporta la información que se le requiere, con sus propias palabras" (Palella y Martins 2012, p.135).

El componente cualitativo se evidencia en la observación de campo donde se prestó atención minuciosa de los diversos grupos, documentación fotográfica y bitácora del desempeño de los estudiantes en la práctica.

La validación se realizó con los estudiantes que matricularon el curso Laboratorio de Zoología en el periodo 2015, participaron un total de 46 personas.

\section{RESULTADOS Y DISCUSIÓN}

La asignatura de Laboratorio de Zoología General que pertenece a la carrera Manejo de Recursos Naturales y el Programa de Producción de Material Audiovisual (PPMA) se plantearon conocer la percepción de los estudiantes respecto al uso y el aporte de los videos didácticos, Especies Marinas: Caribe Submarino y Acuario Marino.

La validación de los audiovisuales tiene por objetivo acercarse a lo que Pablo Freire (2000) llama una educación auténtica donde esta "no se hace de A para B o de A sobre B, sino A con B, con la mediación del mundo. Mundo que impresiona y desafía a unos y a otros originando visiones y puntos de vista en torno de él" (p. 108). 
Algunos de los índices más relevantes a nivel cuantitativo encontrados en la validación que se realizó con los estudiantes que matricularon la asignatura en el periodo del 2015 son:

a. Características de la población estudiantil: participaron del instrumento de validación 46 estudiantes, cuya media de edad fue de 23,8 años de edad.

b. Respecto de la identidad de género, un $60,86 \%$ se identifica con el género femenino y el $39,13 \%$ restante se identifican con el género masculino.

Sobre el video didáctico Especies Marinas: Caribe Submarino destaca la siguiente información:

a. Fue visto por 42 de los 46 estudiantes que participaron del instrumento de validación, equivale al 91,30\%. Esto representa un nivel alto de observación del video como recurso didáctico.

b. Entre la población que manifestó observar el video, 90,75\% se inclinó por las opciones muy clara y clara (ver figura 1) para valorar el nivel de información que les brindó el video con respecto a materiales por utilizar, equipo e indumentaria para la realización del trabajo de campo, anticipar la metodología de la práctica e identificar características y especies marinas y terrestres.

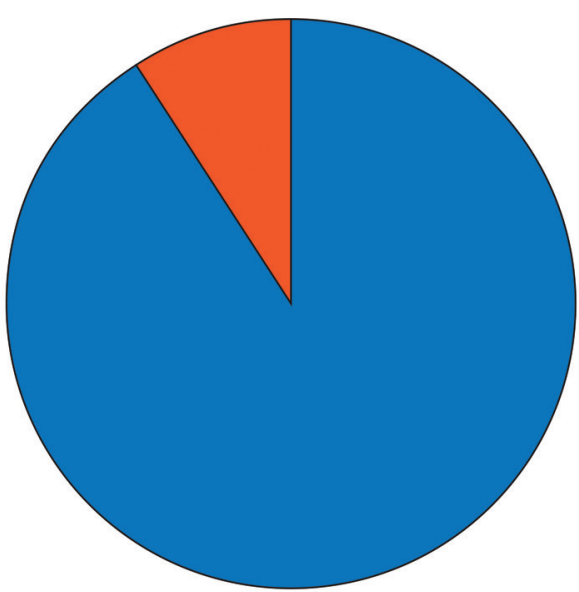

Muy clara y Clara

Figura 1. Video Especies Marinas: Caribe Submarino: Percepción de los estudiantes sobre indumentaria, equipo, metodología e identificación de especies.

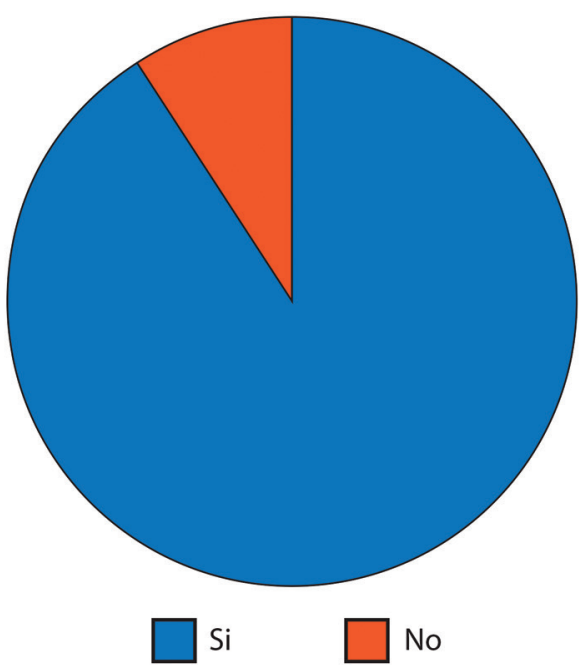

Figura 2. Video Especies Marinas: Caribe Submarino: Percepción de los estudiantes sobre acceso a conocimiento, conceptos y dinámica del trabajo de campo.

c. Entre la población que manifestó observar el video, 91,26\% indicó que sí le permitió ampliar conocimientos y aclarar conceptos o aspectos sobre el tema o contenido de la gira, ver figura 2.

d. En el espacio de consulta abierta llama la atención que $76,19 \%$ de quienes participaron de la validación (tanto quienes lo vieron como quienes no) no respondieron al espacio de observaciones, recomendaciones y sugerencias generales sobre el video didáctico.

e. Sin embargo, entre quienes sí dieron respuesta a la pregunta abierta se encuentran comentarios como los siguientes: "el video es muy claro y preciso con los temas tratados" y "ayudan a entender mejor y a poder visualizar el tema que tratan". 
Sobre el video didáctico Especies Marinas: Acuario Marino destacan las siguientes informaciones:

a. Fue visto por 37 de los 46 estudiantes que participaron del instrumento de validación, equivale a $80,43 \%$. Si bien sigue siendo un porcentaje significativo de visualización es menor al video anterior. Uno de los factores que puede influir en el resultado es que el segundo video se centra en la metodología de intervención en un acuario marino (actividad alternativa para los estudiantes que no pueden asistir a la gira) y no directamente en la práctica de campo; como si ocurre en el primero de los audiovisuales.

b. Entre la población que manifestó observar el video, $88,28 \%$ se inclinó por las opciones muy clara y clara (ver figura 3) para valorar el nivel de información que les brindó el video sobre: identificación y características de especies, metodología de incorporación de individuos al acuario y necesidades de mantenimiento del mismo, así como identificación de especies marinas.

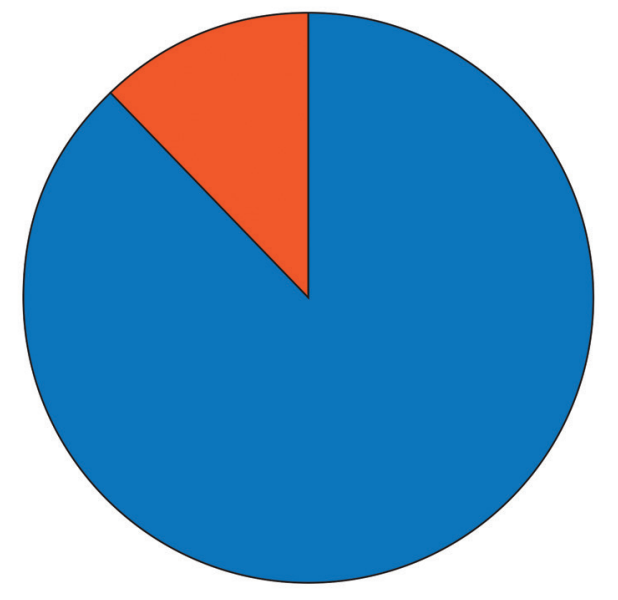

Muy clara y Clara $\square$ Poco clara o deficiente

Figura 3. Video Especies Marinas: Acuario Marino: Percepción de los estudiantes sobre identificación y características de especies, metodología de incorporación de individuos al acuario y necesidades de mantenimiento.

c. Entre la población que manifestó observar el video, 91,89\% indicó que el video sí le permitió ampliar conocimientos, aclarar conceptos y aspectos sobre el tema y lograr una mayor comprensión de los objetivos de la gira o visita al acuario, ver figura 4. De tal manera, se alcanzaron satisfactoriamente los objetivos que guiaron la realización del video.

d. En el espacio de consulta abierta llama la atención que 81,08\% de quienes participaron de la validación (tanto quienes vieron el video como quienes no) no respondieron al espacio de observaciones, recomendaciones y sugerencias generales sobre el video didáctico.

Sin embargo, entre quienes sí dieron respuesta a la pregunta abierta se encuentran comentarios como las siguientes: "el video me fue muy útil para tener mayor conocimiento y estar más preparada en la práctica", "Ios videos son una buena herramienta para estar mejor preparados para la actividad", "todo el video es claro y muy bueno" y "está bien explicado. Me parece que el video está muy bueno". Como parte de las observaciones se destaca "hacer entrevistas a los estudiantes sobre el trabajo realizado", esta última observación cobra gran relevancia por la decisión de la productora y la docente de realizar el video con estudiantes y promover en una próxima producción una puesta en escena que contemple esta variable. 


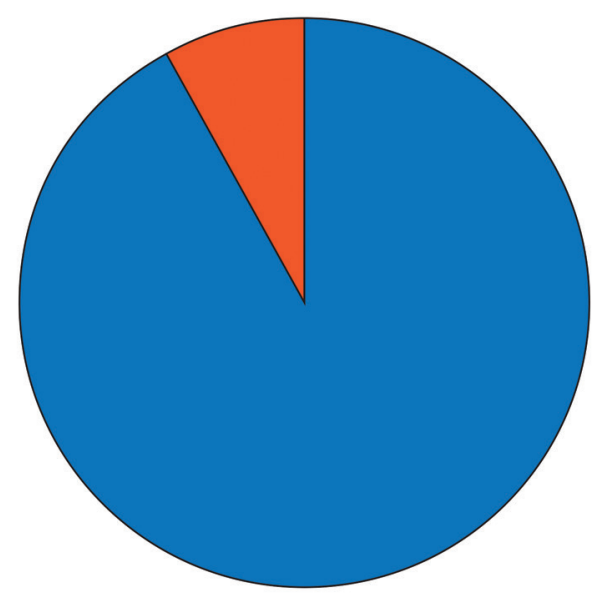

Muy clara y Clara $\square$ Poco clara o deficiente

Figura 4. Video Especies Marinas: Acuario Marino: Percepción de los estudiantes sobre acceso a conocimiento, conceptos y dinámica del trabajo en el acuario.

Los resultados anteriores y específicamente las figuras 1, 2, 3 y 4 muestran que se alcanzaron de manera satisfactoria los objetivos para los cuales se realizó el video.

A nivel cualitativo de observación no participante, se logró evidenciar que el uso de los audiovisuales, previo a la gira, favoreció el desarrollo de las prácticas de los estudiantes en cuanto a vestimenta, equipo y material requerido para el trabajo de campo, facilidad en la dinámica de organización de trabajo grupal y roles por seguir, selección del espacio físico para la ejecución de la práctica, manipulación e identificación de especímenes y sobre todo información teórica y práctica de los contenidos por desarrollar en la gira de campo. En contraste, en años anteriores se había realizado la misma gira de campo sin audiovisuales, por lo que era más complejo la realización de todas las actividades que se desarrollaban, de acuerdo con la experiencia previa que tenían los profesores de la asignatura.

En términos generales, se pudo determinar que los estudiantes no vieron los audiovisuales porque no quisieron o no contaban con el tiempo disponible en el momento que se les solicitaba la visualización (de acuerdo con las razones brindadas en la encuesta de validación que se les aplicó). Sin embargo, entre quienes sí observaron los videos surgen comentarios que promueven el uso de plataformas de distribución como el Youtube y activación de botones de descarga para facilitar el acceso y consumo de los recursos didácticos y que sean compatibles con distintos dispositivos (computadoras, tabletas, celulares) y plataformas como Android e IOS.

Al respecto, la UNED ha puesto a disposición la mayor parte de su producción audiovisual en su propio canal en Youtube (https://www.youtube.com/user/UNEDaudiovisuales) y desde la misma plataforma audiovisuales uned.ac.cr se sugiere el uso de aplicaciones para dispositivos móviles tales como Dolphin, Flash Fox y Puffin.

Si bien existen diversidad de factores que pueden incidir en el índice de aprobación de una asignatura como Laboratorio de Zoología General, se puede señalar como una hipótesis de trabajo para futuras indagaciones el porcentaje de aprobación, donde se puede suponer que los videos didácticos de Especies Marinas lograron incrementar el porcentaje de aprobación de dicha asignatura como se ejemplifica en los siguientes años: para el 2012 y 2013 cuando la asignatura no contaba con los audiovisuales el porcentaje de aprobación fue de 74,3\% y 73, 5\%; mientras que en los años 2014, 2015 y 2016, cuando ya estaba 
la incorporación de estos videos y que representaba el único cambio significativo en la metodología del curso, el porcentaje de aprobación se incrementó a $89 \%, 87 \%$ y $85 \%$ respectivamente. No obstante, se requiere de una adecuada indagación sistemática donde se puedan validar estos datos.

\section{CONCLUSIONES}

La producción de audiovisuales didácticos en la UNED es parte de las estrategias metodológicas que se pueden utilizar pedagógicamente para facilitar una vocación critica, dinámica y creativa que les ayude a los estudiantes a incorporar elementos claves para mejorar su proceso de aprendizaje y que los guíe a promover habilidades y competencias científicas en asignaturas prácticas que involucren las ciencias.

En el caso del video Especies Marinas: Caribe Submarino se tomó la decisión de grabarlo con estudiantes de la asignatura (ellos brindaron su consentimiento); esto con el objetivo de que las generaciones siguientes de estudiantes que se inscriban en la asignatura aprendan de sus pares, compañeros y futuros colegas y también asimilen información previa a la realización del trabajo en el campo. En otras palabras, lo que se requiere es que solos puedan conseguir su autoaprendizaje y así adquirir más competencias del pensamiento científico.

Los dos audiovisuales didácticos de la asignatura, en especial el de Especies Marinas: Caribe Submarino logra incorporar en los estudiantes, una vez que ejecutan la práctica de campo, la aplicación de competencias del pensamiento científico donde alcanzan a desarrollar actitudes, destrezas, habilidades, procedimientos, ser reflexivos, dinámicos y críticos para lograr un individuo autodidáctico y así descubrir soluciones a los problemas que se presentan en los recursos naturales.

Además, por los resultados obtenidos se constata que la producción de videos didácticos requiere una planificación por parte del docente para determinar en cuál momento del cronograma y contenidos de la asignatura ubicar el uso de los videos, con el fin de que su aporte sea pertinente y eficaz.

El proceso de validación de la producción de audiovisuales didácticos con los estudiantes de la carrera MARENA en la asignatura Laboratorio de Zoología General tuvo siempre intrínseco un proceso de diálogo y construcción colectiva que permita mejorar la calidad de la producción didáctica en beneficio de estudiantes, docentes, productores audiovisuales y la institución educativa.

Finalmente, como parte de la validación, uno de los desafíos es cómo interpretar el silencio que se generalizó en las preguntas abiertas, las cuales permitían la posibilidad de plantear observaciones, recomendaciones y sugerencias vinculadas a contenidos, características visuales y sonoras. Sin embargo, los estudiantes omitieron sus comentarios los cuales habrían sido de gran utilidad para este y otros estudios con el fin de priorizar otras variables que favorezcan la calidad de las producciones de videos didácticos.

\section{REFERENCIAS}

Álvarez, P. (2012). Tutoría universitaria inclusiva. Guía de buenas prácticas para la orientación de estudiantes con necesidades educativas específicas. Madrid, Narcea.

Angulo, F. (2012). Evaluar competencias de pensamiento científico en el aula. Directrices metacognitivas. En Quintanilla, M (comp). Las competencias del pensamiento científico desde las voces del aula. (p.p 125-139). Santiago, Editorial Balleterra.

Arias, R. y Vanegas, H. (2013). Caracterización de los procesos de implementación y uso de las aulas virtuales en la Facultad de Educación. Nuevas Ideas En Informática Educ. TISE, (2), 343-352 
Buckingham, D. y Scanlon, M. (2003). Education, Entertainment and Learning in the Home. Buckingham: Open University Press.

Cebrián de la Serna, M. (1994) Los videos didácticos: claves para su producción y evaluación. Revista Pixel Bit, Revista de Medios y Educación, No.1. Recuperado de: http://acdc.sav.us.es/ojs/index.php/ pixelbit/article/view/444/185

De Sousa, B. (2009). Una epistemología del SUR. La reinvención del conocimiento y la emancipación social. México: Siglo XXI \& CLACSO.

Diaz, L; Quintanilla, M y Labarrere, A. (2012). Promoviendo sujetos competentes ante la ciencia y sus problemas. Análisis de microdiseños docentes de evaluación. En Quintanilla, M (comp). Las competencias del pensamiento científico desde las voces del aula. (p.p 83-100). Santiago, Editorial Balleterra.

Field, S. (1996) El Manual del Guionista. Ejercicios e instrucciones para escribir un buen guion paso a paso. Madrid: Plot Edidiones.

Flick, U. (2007). Introducción a la investigación cualitativa. Madrid, Ediciones Morata.

Freire, P. (2000) Pedagogía del oprimido. Edición No. 43. México: Siglo Veintiuno Editores

Palella, S. y Martins, F. (2012) Metodología de investigación cualitativa. Caracas: FEDUPEL.

Quintanilla, M. (2016). Identificación, caracterización y evaluación de competencias científicas desde una imagen naturalizada de ciencia. En Quintanilla y Adúriz-Bravo (eds). Enseñar ciencias en el nuevo milenio. (p.p.17- 42). Santiago, Ediciones PUC.

Ravanal, E. (2012). La noción de metabolismo, como dispositivo epistemológico y didáctico para promover competencias del pensamiento científico. En Quintanilla, M (comp). Las competencias del pensamiento científico desde las voces del aula. (p.p 101-124). Santiago, Editorial Balleterra.

Ruíz, L; Sánchez, M. y Maroto, S. (s.f.). Recursos y materiales en entornos virtuales de aprendizaje. En Consideraciones para el diseño y oferta de asignaturas en línea. (p.p 121-132). Universidad Estatal a Distancia. San José.

Universidad Estatal a Distancia, Costa Rica. (2004). Modelo Pedagógico. Recuperado de http://www. uned.ac.cr/academica/images/igesca/materiales/24.pdf 